\title{
Epidemiology of Cervical Cancer in Taiwan
}

\author{
Pair Dong Wang, M.D., Ph.D., ${ }^{* 1}$ AND Ruey S. Lin, M.D., Dr.P.H. $\dagger$ \\ *Taipei Wanhwa District Health Center, Taipei, Taiwan, Republic of China; and $†$ College of Public Health, \\ National Taiwan University, Taipei, Taiwan, Republic of China
}

Received October 26, 1995

\begin{abstract}
Because the prevalence of several risk factors for cervical cancer, including sexual behavior, reproductive patterns, contraceptive use, and cigarette smoking, has changed significantly over time among Taiwanese women, it is of interest to analyze the impact of these trends on the incidence and mortality rates for this cancer during the decades between 1970 and 1990 . The number of deaths from cervical cancer was obtained from the Taiwan Provincial Department of Health during the period from 1974 to 1992. Data of all incident cervical cancer cases from 1979 through 1990 obtained from the Taiwan Cancer Registry were also used for the analyses. The trends in the age-adjusted and age-specific rates were examined. Age, period, and cohort effects on the incidence and mortality rates were also examined, using age- period-cohort (APC) models. The age-adjusted mortality rate has been increasing since the 1970s, particularly in both the youngest group (20-29 years) and the oldest group (65 years and over). While the ageadjusted incidence rate decreased from 1979 to 1990, and decreases were apparent across all broad age groups, nevertheless, the incidence of adenocarcinoma was moderating during the same time period. The APC models disclosed a decreasing cohort effect for the mortality and incidence rates among women born after 1936. Decreasing incidence rates after menopause were more consistent with a hormonal effect than a cohort effect. D eclining trends in cervical cancer incidence may be related to the screening practices which may have counteracted the increasing prevalence of several risk factors, although poor survival influenced the incidence/mortality differentials for cervical cancer. Continued surveillance is warranted; however, attention might be directed to the trends in cervical adenocarcinoma. 1996 Academic Press, Inc.
\end{abstract}

\section{INTRODUCTION}

Although during the past several decades, the rate of cervical cancer have declined significantly in Western countries, it remains one of the most common female forms of cancer in developing countries [1]. In Taiwan, as recent as the early 1990s cervical cancer still remained the most frequent neo-

\footnotetext{
${ }^{1}$ To whom correspondence and reprint requests should be addressed at Wanhwa District Health Center, No. 152, Tung-Yuan Street, Taipei, Taiwan, Republic of China. Fax: 886-2-3323514.
}

plasm, accounting for more than $30 \%$ of all cancer forms within this population [2]. The annual age-adjusted mortality rate of cervical cancer in Taiwan increased from 6.06 per 100,000 in 1974 to 10.02 in 1993, a 1.4-fold increase over 20 years [3]. This malignancy is now the lead in cancer incidence and is the third leading cause of cancer deaths among Taiwan's females, with a cumulative incidence and mortality rate to age 85 of 4.2 and $1.3 \%$, respectively [2].

The trend in the mortality and incidence of this cancer may be potentially influenced by exposures to changing environmental factors. Since temporal factors (e.g., age, period, and birth cohort) are particularly sensitive indicators of a changing environment, we have attempted to describe the nature and timing of changes in cervical cancer incidence and mortality to define when and where etiologic clues to this trend may be found and to help assess the effects of improved screening and measures of intervention.

\section{MATERIALS AND METHODS}

\section{Mortality Data and Population}

Information on all reported mortality cases in Taiwan from 1974 to 1992 was abstracted from the annual reports published by the Taiwan Provincial Department of Health. Cervical cancers referenced in this study were defined as code 180 in the International Classification of Disease, 8th and 9th revision (ICD-8 and ICD-9). Age-specific midyear population estimations in Taiwan from 1974 to 1992 were obtained from data published by the Ministry of the Interior in Taiwan [4]. Mortality rates were stratified by 5-year age groups.

\section{Incidence Data}

The National Cancer Registry Campaign was begun only recently in Taiwan and hence information about the incidence of cervical cancer since 1979 was available for this study. Data of all incident cervical cancer cases from 1979 through 1990 were obtained from the Taiwan Cancer Regis- 


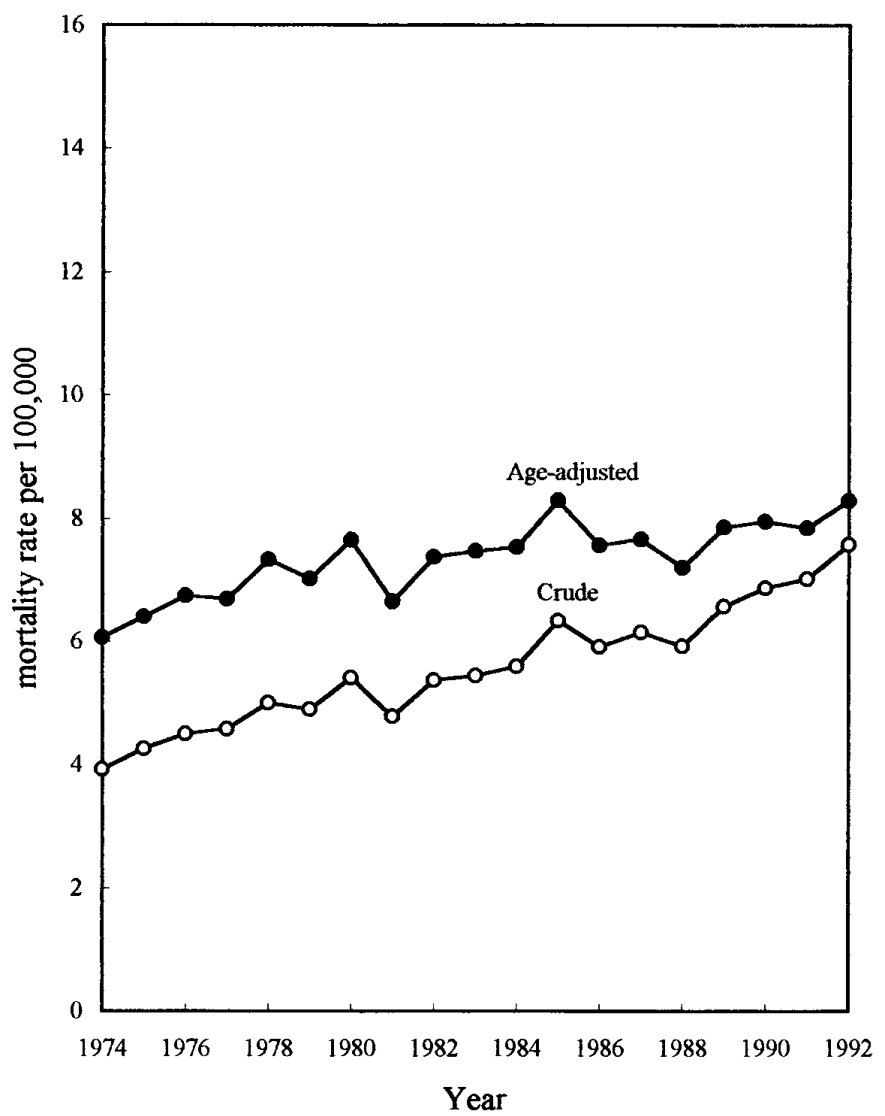

FIG. 1. Trends in crude and age-adjusted mortality rates of cervical cancer in Taiwan from 1974 to 1992.

try [2]. Each cervical cancer case was characterized by detailed demographic data including age at diagnosis, period at diagnosis, and birth date. Cervical cancers reported to Taiwan Cancer Registry were classified into three histologic types according to the International Classification of Disease for Oncology (ICD-0) [5]: squamous cell carcinoma (ICD0 codes 8000, 8010, 8070-8072, and 8076), adenocarcinoma (ICD-0 codes 8140, 8260, 8430, 8441, 8480, 8481, and 8560), and adenosquamous cell carcinoma (ICD-0 codes 8560 and 8570). Cases for which the cervix histologic type could not be determined (ICD-0 code 9990) were also tabulated (cervix, not otherwise specified). A total of 21,726 women with cervical cancer was collected for the analyses during the period 1979-1990. Incidence rates were stratified by 3 -year age groups.

\section{Descriptive Analysis}

To adjust for the effect of difference in age composition for different periods, the total and age-specific rates of cervical cancer mortality from 1974 to 1992 and incidence from 1979 to 1990 in Taiwan were age-adjusted to the 1976 world population [7].

\section{Statistical Age-Period-Cohort Analysis}

The matrix of the age-specific death rate was calculated for each 5-year period interval, beginning with 1974 to 1978 , and 5-year age interval, beginning with age 20 to 24 . The matrix of the age-specific incidence rates was calculated for each 3-year period interval, beginning with 1979 to 1981, and 3-year age interval, beginning with age 25 to 27 . The effects of age, period, and birth cohort were examined using a log-linear Poisson regression model, modified from Osmond and Gardner's method [7]. The statistical model used in these analyses was

$$
\log \left(R_{\mathrm{ijk}}\right)=K+A_{\mathrm{i}}+P_{\mathrm{j}}+C_{\mathrm{k}}+E_{\mathrm{ijk}},
$$

where $R_{\mathrm{ijk}}$ represents the observed mortality/incidence rate in a particular age-period-cohort category; $K$ is a constant; $A_{\mathrm{i}}, P_{\mathrm{j}}$, and $C_{k}$ represent the age, period, and cohort effects, respectively; and $E_{\mathrm{ijk}}$ represents random error. The mortality/ incidence from cervical cancer is assumed to follow a Poisson distribution.

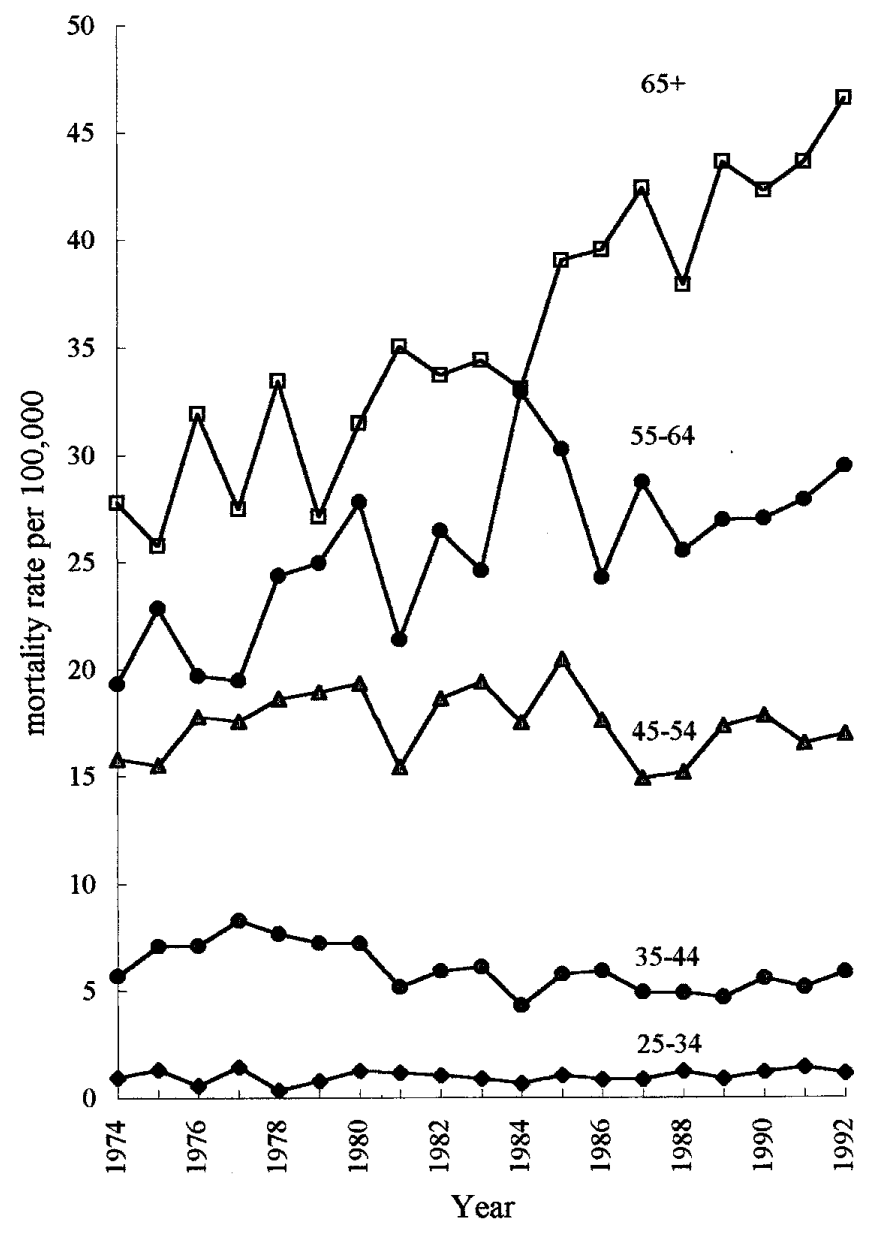

FIG. 2. Trends in age-specific mortality rates of cervical cancer in Taiwan, 1974-1992. 


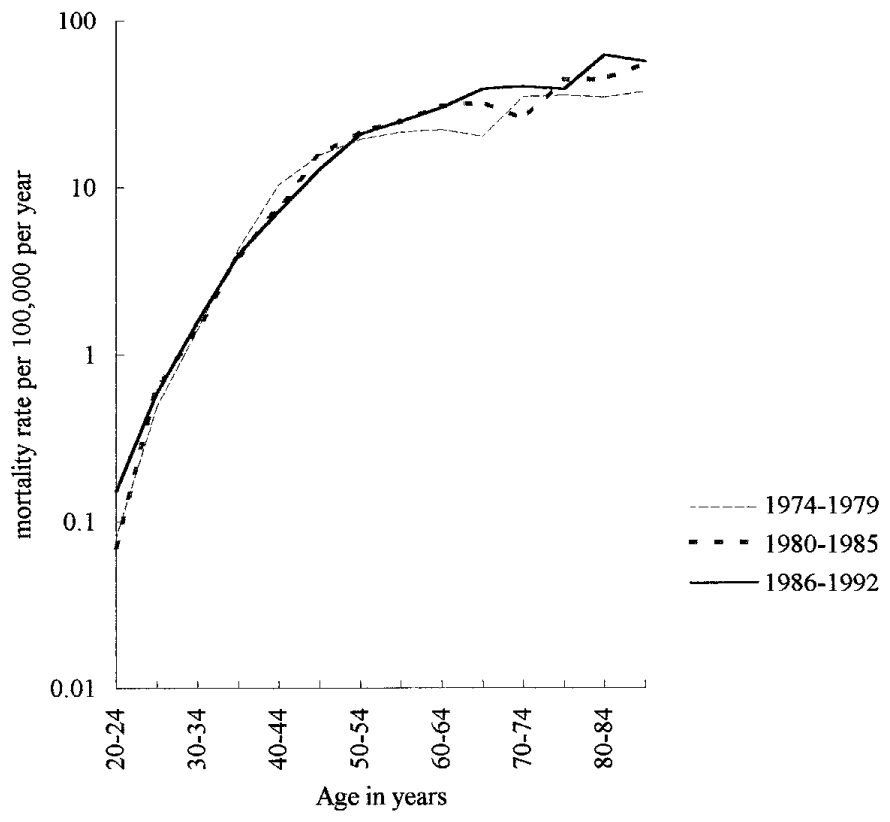

FIG. 3. Age-specific mortality rates of cervical cancer in Taiwan from 1974 to 1992.

The estimates derived from the model, including the three time factors, that minimized the weighted sum of the Euclidean distances from the three possible two-factor models (age/period, age/cohort, and period/cohort) were based on the goodness-of-fit of each one. In the study,

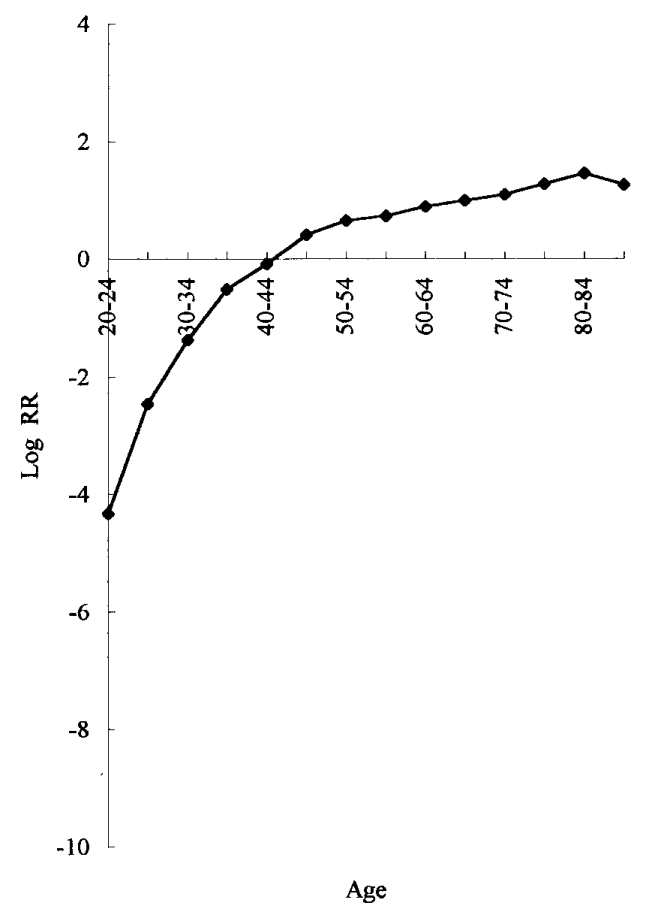

FIG. 4. Age effects on cervical cancer mortality in Taiwan from 1974 to 1992 .

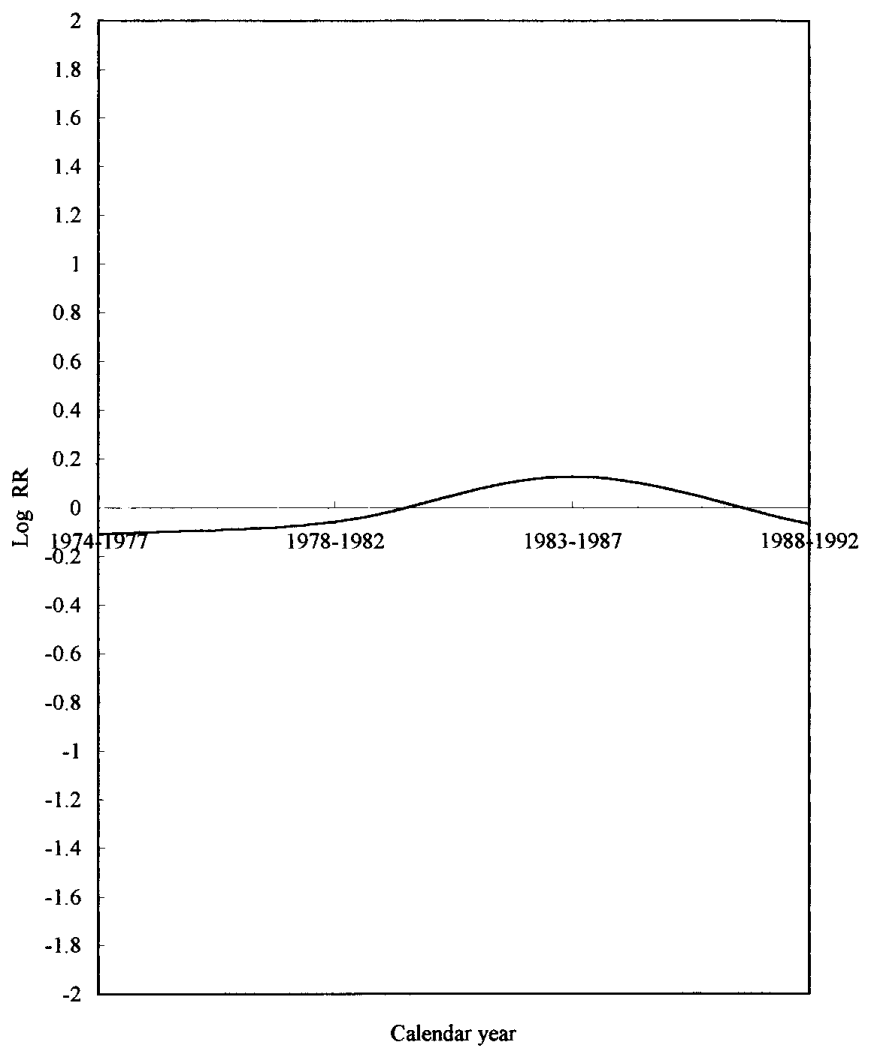

FIG. 5. Period effects on cervical cancer mortality in Taiwan from 1974 to 1992 .

these measures were taken as the inverse of the deviance statistics. The sum of each of the three effects was constrained to be zero. These effects can be interpreted as logarithms of relative risks. The computer program SAS/ IML was used for the computation [8].

\section{RESULTS}

\section{Cervical Cancer Mortality}

Figure 1 shows the trends of crude, age-adjusted, and cumulative mortality rates of cervical cancer in Taiwan between 1974 and 1992 . The crude mortality rate was 3.93 per 100,000 in 1974 and 7.58 in 1992. The age-adjusted mortality rate was 6.06 per 100,000 in 1974 and 8.28 in 1992. The average annual increase of cervical cancer mortality rate was $0.09 \%$ during this study period.

Trends in the age-specific mortality rates for each 10-year age group are plotted in Fig. 2. The upward trend in mortality was evident primarily at the age groups of 20-29 and 65 years and older. This observation can be also noted in Fig. 3 , which represents the age-specific death rates plotted against age. Within the three time periods, the age-specific mortality sharply increased until the age group of 50-54 years, with a smaller slope up to the age of 85-89 years. 


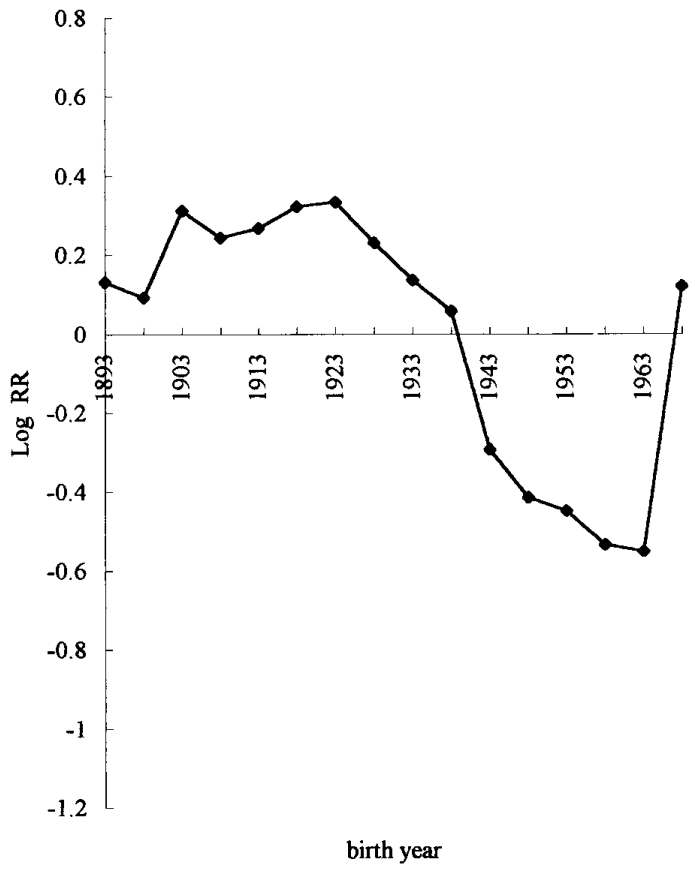

FIG. 6. Cohort effects on cervical cancer mortality in Taiwan from 1974 to 1992.

However, this analysis of age-specific death rates did not consider the period and cohort effect upon which the secular trend depended.

Figure 4 shows the logarithm of relative risks of cervical cancer mortality in different age groups (i.e., the age effects) as derived from the age-period-cohort (APC) model. The age effects increased with age even after menopausal age, although the slope became much more moderate, corresponding to menopausal age. The older the age, the greater the risk. The older age group of 50-54 years has 89.3 times the risk of the younger age group of 30-34 years.

Figure 5 shows the period effects. The period effect increased from 1974-1977 to 1973-1987 and then declined slightly. The period effect was less striking than the age effect.

The cohort effect is depicted in Fig. 6. A steady decrease in the cohort effect was observed among women born after 1938, except for an increase in the birth cohort of 1968.

\section{Cervical Cancer Incidence}

Crude, age-adjusted, and cumulative incidence rates of cervical cancer in Taiwan between 1979 and 1990 are shown in Fig. 7. It can be seen that the crude incidence rate has decreased from 20.81 per 100,000 in 1979 to 17.35 in 1990 and the age-adjusted incidence rate from 28.43 per 100,000 to 19.45 in 1990 . The average annual decrease of incidence rate was $0.88 \%$ during this study period.

Trends in the age-specific incidence rate are shown in Fig. 8. The rate has rapidly decreased, particularly in the age

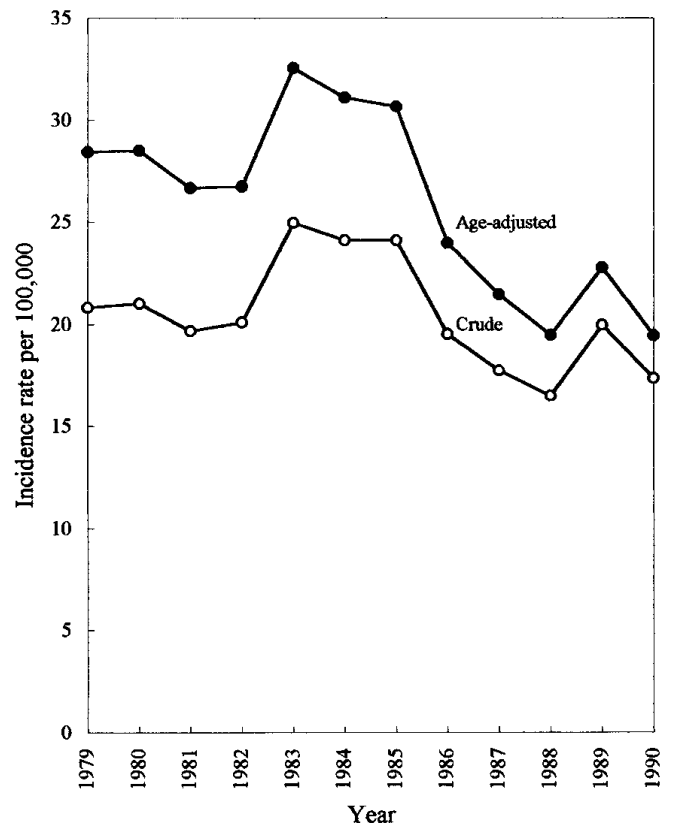

FIG. 7. Trends in crude and age-adjusted incidence rates of cervical cancer in Taiwan from 1979 through 1990.

range of 35-54 years. Figure 9 plots age-specific incidence against age. In each time period, that is, in 1979-1981, 1982-1984, 1985-1987, and 1988-1990, the rate rose ex-

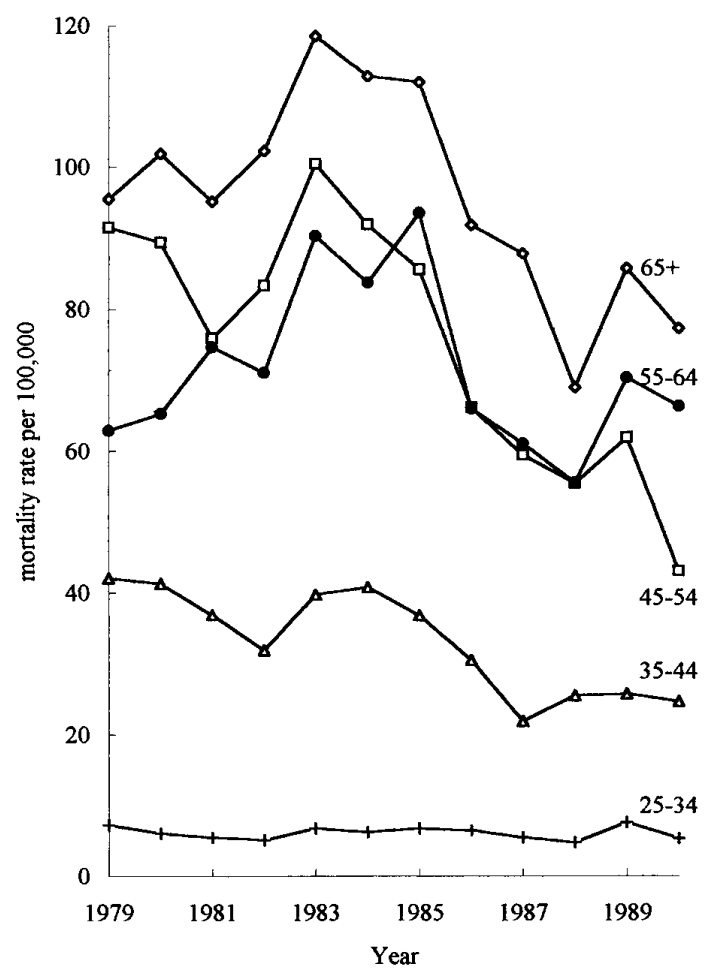

FIG. 8. Trend in age-specific incidence rates of cervical cancer in Taiwan, 1979-1990. 
ponentially up to the age group of 52-54 years and then declined with advancing age.

Figure 10 shows the age effects on incidence rates of cervical cancer. Prior to the age group of 49-51 years, there is a near linear trend in the effect of age in the form of the logarithm of relative risks for cervical cancer, after which it decreases with age. Figure 11 shows the period effect, also presented in the form of the logarithm of relative risks. A slight increase was evident from 1979-1981 to 19821984 and then declined consistently. Figure 12 shows the cohort effect from 1909 to 1960 . For the earlier birth cohorts, the cohort effect fluctuates and remains above the average, while the cohort effect has dramatically decreased after the birth cohort of 1936, with the exception of a slight increase in the birth cohort of 1951 and 1957. The decrease was such that the 1936 cohort had a risk of cervical cancer 3.8 times larger than the 1960 cohort.

\section{Cervical Cancer Incidence by Histologic Type}

Table 1 shows the rates of cervical cancer by age, time period, and histologic type. While the data show that the incidence rates of squamous cell carcinoma decreased in all age groups, adenocarcinoma, however, increased from 1.19

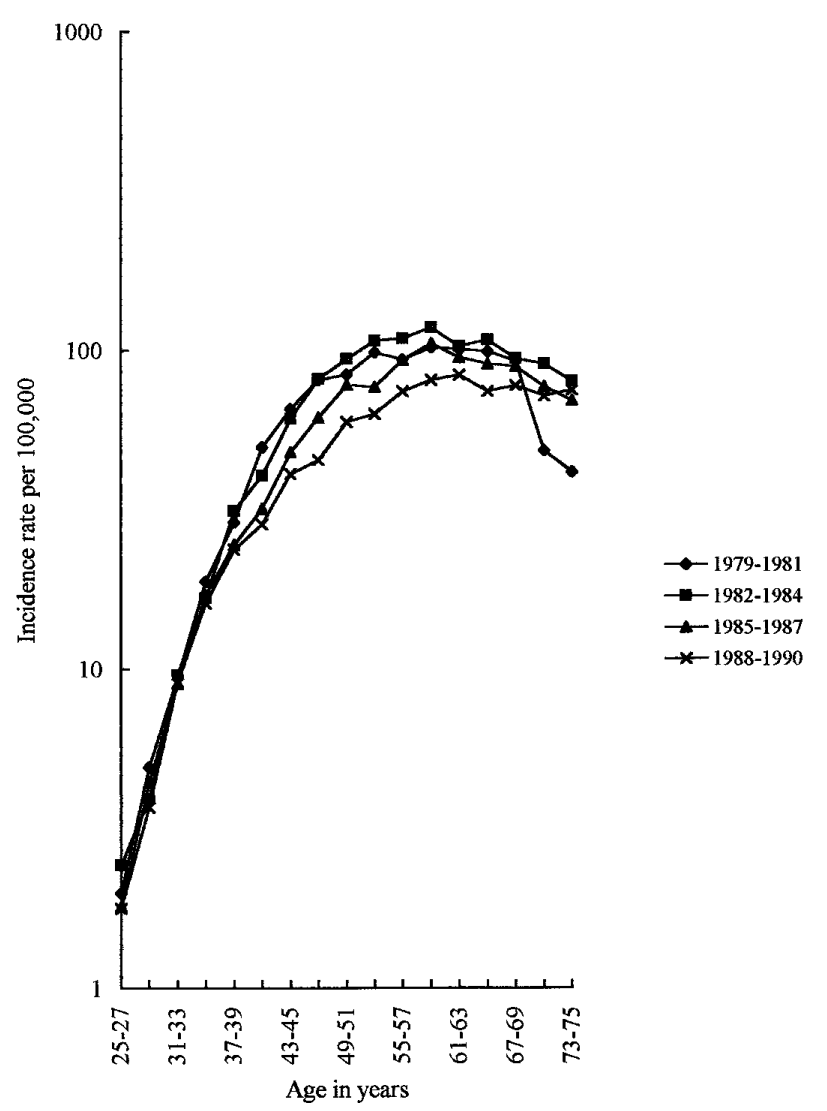

FIG. 9. Age-specific incidence rates of cervical cancer in Taiwan from 1979 through 1990.

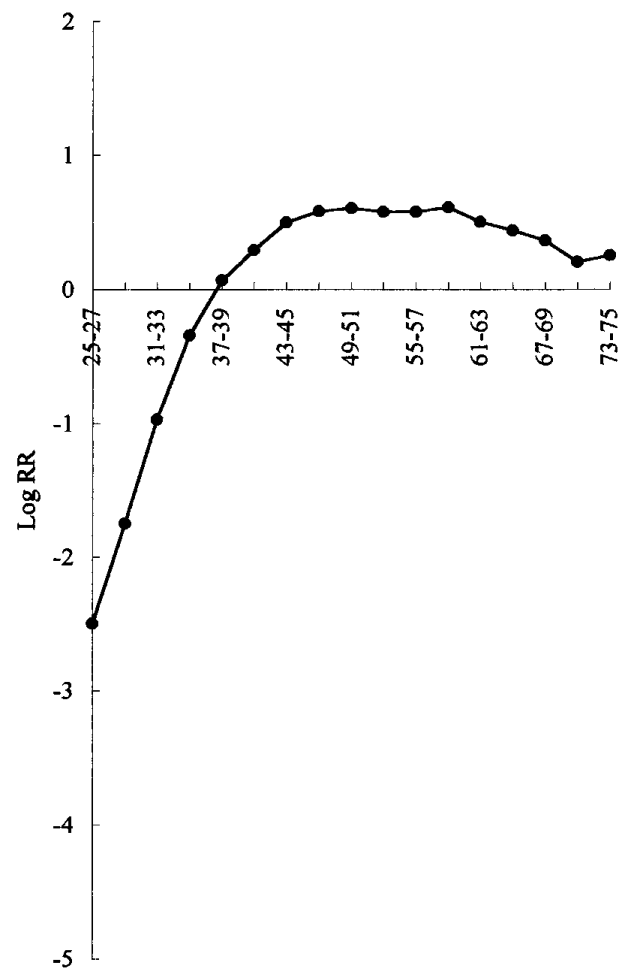

Age

FIG. 10. Age effects on the incidence of cervical cancer in Taiwan between 1979 and 1990 .

to 1.41 per 100,000 between 1979 and 1990 , with the increase being more pronounced within the age group of 2534 years. Adenosquamous carcinomas are even less common, and rates change little after increasing during the period of 1979-1982 to 1983-1986. The rates of invasive carcinoma that were not further specified by histologic type decreased from 1979-1982 to 1983-1986 and then plateaued.

\section{DISCUSSION}

In the present study, we have summarized the trends in cervical cancer mortality and incidence rates in Taiwan. Most notable among these trends, the age-adjusted incidence rate decreased by $31.6 \%$ between 1979 and 1990, while the age-adjusted mortality rate increased by $15.2 \%$. This disparity reflects a poor survival rate of cervical cancer in Taiwan. However, in recent years, the cervical cancer incidence rates have been clearly declining and may reflect in part the increasing use of the Pap smear [9]. This trend may ultimately lead to a decline in cervical cancer mortality, although mortality trends can be expected to lag behind incidence variations in view of the fact that the median survival time for women diagnosed with cervical cancer is about 6 years in Taiwan [9]. From the data presented in Table 1 the incidence of adenocarcinoma increased from 1.19 to 1.41 per 


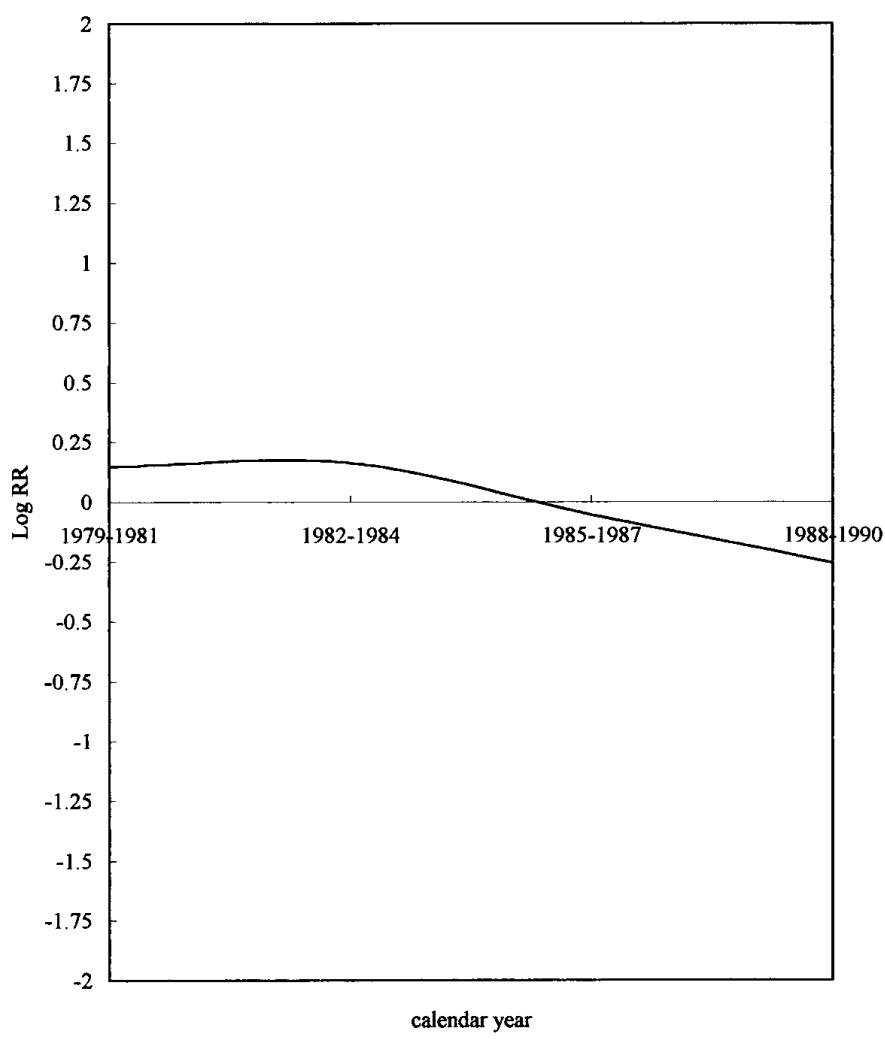

FIG. 11. Period effects on the incidence of cervical cancer in Taiwan between 1979 and 1990 .

100,000 between 1979 and 1990, compared with a significant decrease in the squamous cell carcinoma, the increase being more pronounced among the age group of 25-34 years. This pattern is consistent with several studies from different geographical areas, including the U.S.A. [10], UK [11], and Norway [12].

Time trend of mortality and incidence rates for a particular disease can provide an epidemiologist with valuable clues or hypotheses for disease etiology [13]. Three temporal factors which are often considered in such an investigation are age, time period, and birth cohort. In 1939, Frost first employed these three factors on mortality rates from tuberculosis in Massachusetts [14]. This technique was adopted further by Case to establish the value of cohort analysis [15], but the technique remained a graphical one and the contributions of various time factors were determined visually to examine patterns in disease rates over time [16]. In contrast, statistical APC analysis has been developed in an attempt to overcome these drawbacks and to quantify the separate effects of the age, period, and cohort variables, provided suitable constraints are imposed [17]. The constraints proposed by Osmond and Gardner were determined solely within the data and yielded an objective indication of the statistical significance of a particular pattern [7]. We used the APC model for two goals: (1) to determine in which generations the decreasing trends in mortality and incidence began and (2) to quantify the separate effects of the age, period, and cohort variables on the mortality and incidence rates, adjusting for the temporal effects. The model adequately addresses these purposes, because it derives the separate effects of age, period, and cohort on mortality and incidence rates adjusted for one another. Although the limitation of this technique was subsequently noted by Holford, and a more objective method among the APC models proposed [13], the analyses described the present data very well and successfully attained the aims mentioned above.

Most epidemiologic studies have incriminated various risk factors for cervical cancer. Major risk factors for cervical cancer include multiple sex partners and early age at first intercourse $[18,19]$. However, the sexual behavior of Taiwanese men and women differs from contemporary Western practices. There is a conventional double standard for sexual behavior in Taiwan's population. While female virginity prior to marriage and fidelity subsequent to marriage are highly valued, many husbands frequently visit prostitutesa practice which is tolerated within society. Given this pattern of sexual behavior, prostitutes may act as an infectious reservoir, and the husband's sexual behavior may be a major determinant in the risk factors for cervical cancer in wives, particularly among those not using condoms. This idea was supported by Slattery et al., who postulated that the regular use of condoms may protect the cervix from opportunistic transmission by infectious agents [20]. Our previous casecontrol study [21] conducted in Taiwan during the period 1991-1994 revealed that the mean lifetime number of sex partners slightly increased from an average of 1.1 among women age 50 and older to 1.5 among those under age 40 . Furthermore, the average age at first intercourse declined from 23.7 to 20.4, and the percentage of a husband's ever visiting prostitutes increased from 28.6 to $40.4 \%$. Moreover, the prevalence of condom use has been less than $18 \%$ of all women. However, the protective role played by condoms has probably been minor due to the low prevalence of use in Taiwan, although usage is now increasing in response to the AIDS epidemic. These differences partly reflect the increasing sexual freedom arising within the past several decades, and it is supported by the parallel trend rates of sexually transmitted diseases [22]. Yet changes in sexual activity are not reflected in the trends for cervical cancer.

Recent studies among Hispanic women in the U.S.A. and in Latin America [23, 24], in which multiple births are more common, indicate that the number of births has an independent and relevant role in cervical cancer. In Taiwan, the total fertility rate fell rapidly from 1960 to 1980 : 5.78 in 1960 and 2.50 in 1980 [3]. The rate was almost fixed at 2.50 in the early 1980 s and then showed a gradually declining trend, and in 1992 it was 1.80 . The fact that these findings reflect a rapid decrease in the cohort effect on incidence after the 1936 birth cohort (Fig. 12) might be partly explained by the 
TABLE 1

Incidence $^{a}$ of Cervical Cancer by Histologic Type, Age, and Time Period, Taiwan Areas, 1979- 1990

\begin{tabular}{|c|c|c|c|c|c|}
\hline \multirow[b]{2}{*}{ Time period and histologic type } & \multicolumn{5}{|c|}{ Incidence (no. of cases) } \\
\hline & $25-34$ & $35-49$ & $50-64$ & $65+$ & All ages \\
\hline \multicolumn{6}{|l|}{ Squamous cell carcinoma } \\
\hline $1979-1982$ & $1.23(301)$ & $42.61(2134)$ & $83.86(2628)$ & $56.76(869)$ & $17.41(5932)$ \\
\hline $1983-1986$ & $1.56(399)$ & $42.57(2347)$ & $90.73(3283)$ & 70.52 (1269) & $20.03(7298)$ \\
\hline $1987-1990$ & $1.36(346)$ & $25.01(1600)$ & $61.63(2531)$ & $51.95(1121)$ & $14.74(5658)$ \\
\hline & $5.7^{b}(0.02 *)$ & $87.80(0.00 *)$ & $98.70(0.00 *)$ & $4.60(0.03 *)$ & $39.50\left(0.00^{*}\right)$ \\
\hline \multicolumn{6}{|l|}{ Adenocarcinoma } \\
\hline 1979-1982 & $0.14(34)$ & $3.23(162)$ & $4.98(156)$ & $3.40(52)$ & $1.19(404)$ \\
\hline $1983-1986$ & $0.22(56)$ & $3.45(190)$ & $5.97(216)$ & $5.22(94)$ & $1.53(556)$ \\
\hline $1987-1990$ & $0.23(57)$ & $2.79(185)$ & $5.65(232)$ & $3.06(66)$ & $1.41(540)$ \\
\hline & $4.76(0.05 *)$ & $2.20(0.14)$ & $1.20(0.28)$ & $0.7 \quad(0.40)$ & $5.4(0.02 *)$ \\
\hline \multicolumn{6}{|l|}{ Adenosquamous carcinoma } \\
\hline $1979-1982$ & $0.01(2)$ & $0.38(19)$ & $0.51(16)$ & $0.26(4)$ & $0.12(41)$ \\
\hline $1983-1986$ & $0.04(9)$ & $0.76(42)$ & $1.22(44)$ & $0.56(10)$ & $0.29(105)$ \\
\hline $1987-1990$ & $0.05(12)$ & $0.66(44)$ & $1.07(44)$ & $0.70(15)$ & $0.30(115)$ \\
\hline & $6.1 \quad(0.02 *)$ & $3.2(0.08)$ & $5.1(0.02 *)$ & $3.1(0.08)$ & $23.4\left(0.00^{*}\right)$ \\
\hline \multicolumn{6}{|l|}{$\begin{array}{l}\text { Other and unspecified epithelial } \\
\text { cancer }\end{array}$} \\
\hline $1979-1982$ & $0.14(33)$ & $3.53(177)$ & $7.37(231)$ & $8.23(126)$ & $1.66(567)$ \\
\hline $1983-1986$ & $0.13(34)$ & $2.21(122)$ & $5.47(198)$ & $6.72(121)$ & $1.30(457)$ \\
\hline \multirow[t]{2}{*}{$1987-1990$} & $0.17(43)$ & $2.09(139)$ & $5.14(211)$ & $7.74(167)$ & $1.46(560)$ \\
\hline & $1.0 \quad(0.32)$ & $21.6(0.00 *)$ & $14.2(0.00 *)$ & $0.14(0.71)$ & $4.7(0.03 *)$ \\
\hline
\end{tabular}

\footnotetext{
${ }^{a}$ Rate per 100,000 women per year.

${ }^{b} \chi^{2}$ value for trend test ( $P$ value).

* Significant at $\alpha=0.05$ level.
}

decrease in parity, since the 1936-1950 birth cohort, in our analysis, came into child-bearing age in the midst of 1960s1980s, which was a period of family planning and a marked birth control campaign in Taiwan. This coincidence in time may imply that reproductive factors are one of the most possible sources of the cohort effect.

It has been suggested that the increased use of OCs may be linked to the increase in cervical cancer incidence [28], while Winkelstein found in 15 of 18 studies carried out since 1966 an association of smoking with the increased risk of cervical cancer [27]. Yet the prevalence of Taiwanese women who ever smoked cigarettes is as low as $3.2 \%$, and the prevalence of those who ever used oral contraceptives is $8.7 \%$. Thus oral contraceptives and smoking explain little of the time trends in cervical cancer among this population.

An important factor influencing cervical cancer trends is the increased use of screening practices to detect precursor lesions while reducing the incidence of invasive cervical cancer $[25,26]$. Our previous study showed that the percentage of women older than 30 who reported ever having had a Pap smear increased from $23.5 \%$ in 1970 s to $63.9 \%$ in 1990s [9]. These patterns are consistent with the observed trends in cervical cancer incidence rates (Fig. 7). However, Taiwan data contrast with those of the UK, where an increase in cervical cancer incidence has been reported at young ages [29].
In the age-mortality curve in Taiwan, 1974-1992, the rates increased with age even after so-called age at menopause (Fig. 3); however, in the age-incidence curve in Taiwan, 1979-1990, a small decline with age was noted after menopause (Fig. 9). Our analysis using the APC mode provides clue to rule out this fall in cross-sectional rates after menopause to a cohort effect, because the age effect on incidence, which is adjusted for the cohort and period effect, also decreases after the age at menopause. Thus it is reasonable to suggest that occurrence of cervical cancer may be influenced by female sex hormones such that incidence stops linear increase after the menopause. This explanation is consistent with the patterns observed elsewhere, including the UK, Finland, and Norway [30].

In applying to APC analyses to mortality and incidence trends in the present study, there was a substantially decreasing risk of cervical cancer incidence during the period from 1979-1981 to 1988-1990, while the period effect on mortality was moderating during the period from 1974-1977 to 1988-1992. Interpretation of these findings is complicated by the fact that the period effects could be due to factors such as changes over time in medical care, screening practices, prevalence of hysterectomy, and registration practices. Thus, it is possible that period effects do not reflect etiologic distinctions, but more trivial period factors.

As regards cohort effects, for the earlier birth cohorts, the 


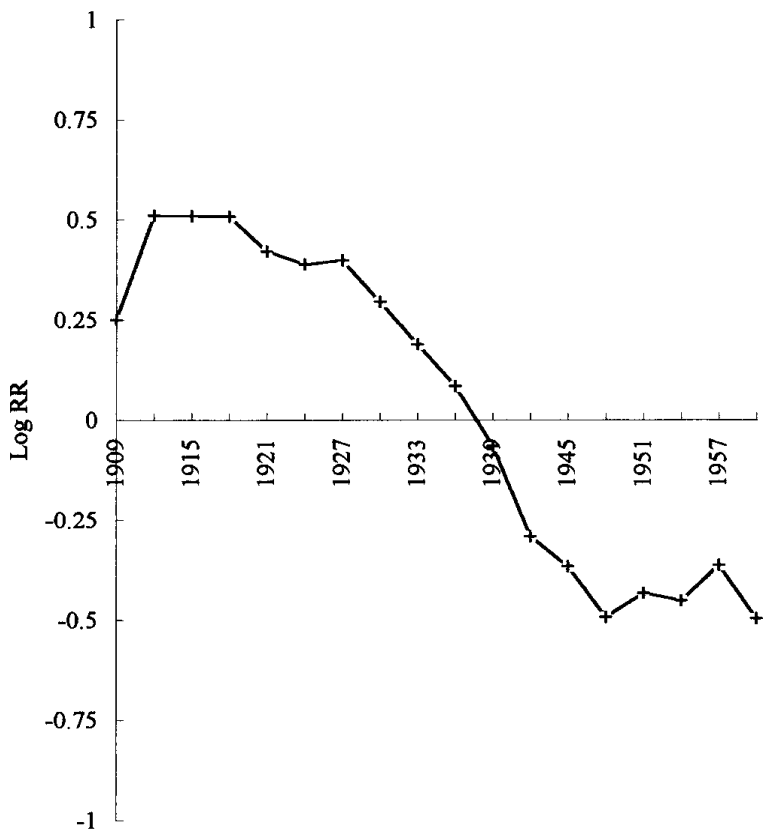

birth year

FIG. 12. Cohort effects on the incidence of cervical cancer in Taiwan between 1979 and 1990 .

cohort effect fluctuates and remains above the average, while the relative risks decrease sharply after the 1938 birth cohort for mortality and the 1936 birth cohort for incidence of cervical cancer. However, the risk increased on mortality in the 1968 cohort and remained at a plateau on incidence in the 1951-1957 cohort. This phenomenon suggests that generations of women born in 1951-1957 and 1968 have been increasingly exposed to a cause of cervical cancer. It may reflect in our analysis that in the most recent time period, there has been an upward trend in mortality in these young women (Fig. 2). These age-related variations highlight the urgent need for improvements in screening strategies among this age group in Taiwan.

Some perspectives on the trend in cervical cancer incidence were noted in Fig. 8. The incidence rates in those age $\geqslant 55$ years have not notably decreased. However, the incidence in this age group will be expected to decrease substantially when the 1936-1950 birth cohort (the incidence has decreased rapidly during this cohort) reaches the age of 55 years in the 2000s.

In summary, it appears that the decline in cervical cancer incidence rates, in spite of the increasing prevalence of some risk factors, might reflect increases in the level of screening activities in Taiwan. However, the mortality rates did not decrease, partly due to the poor survival rate. In the future, further incidence declines may be possible with the 19361950 cohort reaching the age 55 years and older in the 2000s and with the usage of screening gradually increasing with time. Although increases in adenocarcinoma during 1982-
1984 appeared to be moderating during 1988-1990, continued surveillance is warranted.

\section{ACKNOWLEDGMENTS}

We thank Dr. J. M. Weaver for many valuable comments and Mr. W. T. Ho for technical assistance.

\section{REFERENCES}

1. Ponten, J., Adami, H. O., Bergstrom, R., Dillner, J., Friberg, L. G., Gustafsson, L., Miller, A. B., Parkin, D. M., and Trichopoulos, D. Strategies for global control of cervical cancer, Int. J. Cancer 60, $1-$ 26 (1995).

2. Department of Health, the Executive Yuan. Cancer registry annual report in Taiwan area 1979-1990, Department of Health, Executive Yuan, Taipei (1980-1991).

3. Taiwan Provincial Department of Health. Vital statistics, 1960-1993. Taiwan Provincial Department of Health, Chung-Hsing Village (19611994).

4. Ministry of the Interior, R.O.C. Demographic Facts 1974-1992, Ministry of Interior, R.O.C., Taipei (1975-1993).

5. World Health Organization, ICD-O. International classification of diseases for oncology, WHO, Geneva (1976).

6. Smith, P. G. Comparison between registries: Age-standardized rates, in (D. M. Parkin, C. S. Muir, S. L. Whelan, Y. T. Gao, J. Ferlay, and J. Powell, Eds.), International Agency for Research on Cancer, Lyon, pp. 865-870 (1992).

7. Osmond, C., and Gardner, M. J. Age, period and cohort models applied to cancer mortality rate, Stat. Med. 1, 245-259 (1982).

8. SAS Institute Inc. SAS/IML: User's guide, release 6.04 ed., SAS Institute, Inc. Cary, NC (1988).

9. Wang, P. D., and Lin, R. S. Cervical cancer screening in Taiwan: Fiveyear results, Chin. Med. J. 109(4), 286-290.

10. Devesa, S. S., Young, J. L., Brinton, L. A., and Fraumeni, J. F. Recent trends in cervix uteri cancer, Cancer 64, 2184-2190 (1989).

11. Chilvers, C., Mant, D., and Pike, M. C. Cervical adenocarcinoma and oral contraceptives, Br. Med. J. 295, 1446-1447 (1987).

12. Eide, J. Cancer of uterine cervix in Norway by histologic type, 197084, J. Natl. Cancer Inst. 79, 199-203 (1987).

13. Holford, T. R. The estimation of age, period and cohort effects for vital rates, Biometrices 39, 311-324 (1983).

14. Frost, W. H. The age selection of mortality from tuberculosis in successive decade, Am. J. Hyg. 30, 91-96 (1939).

15. Case, R. A. M. Cohort analysis of mortality rates as a historical or narrative technique, Br. J. Prev. Soc. Med. 10, 159-171 (1956).

16. MacMahon, B., and Terry, W. B. Application of cohort analysis to the study of time trends in neoplastic disease, J. Chronic Dis. 7, 24-35 (1958).

17. Kupper, L. L., Janis, J. M., Darmous, A., and Greenberg, B. G. Statistical age-period-cohort analysis: A review and critique, J. Chronic Dis. 38, 811-830 (1985).

18. Brisson, J., Morin, C., Fortier, M., Roy, M., Bouchard, C., Leclerc, J., Christen, A., Guimont, C., Penault, F., and Meisels, A. Risk factors for cervical intraepithelial neoplasia: Differences between low- and high-grade lesions, Am. J. Epidemiol. 140, 700-710 (1994).

19. Armstrong, B., Munoz, N., and Bosch, F. X. Epidemiology of cancer of the cervix, in Gynecologic Oncology, 2nd ed. (M. Coppleson, M. J. Monaghan, C. P. Morrow, and M. I. N. Tattershall, Eds.), Churchill Livingstone, Edinburgh, pp. 11-29 (1992). 
20. Slattery, M. L., Overall, J. C., Abbott, T. M., French, T. K., Robinson, L. M., and Gardner, J. Sexual activity, contraception, genital infections, and cervical cancer: Support for a sexually transmitted disease hypothesis, Am. J. Epidemiol. 130, 248-257 (1989).

21. Wang, P. D., and Lin, R. S. Risk factors for cervical intraepithelial neoplasia in Taiwan, Gynecol. Oncol. 62, 10-18 (1996).

22. Venereal Disease Center, Health Department of Taipei City Government. Sexually transmitted disease annual report, 1993. Venereal Disease Center, Health Department of Taipei City Government, Taipei (1994).

23. Brinton, L. A., Reeves, W. C., Brenes, M. M., et al. Parity as a risk factor for cervical cancer, Am. J. Epidemiol. 130, 486-496 (1989).

24. Herrero, R., Brinton, L. A., Reeves, W. C., Brenes, M. M., De Brinton, R. C., Gaitan, E., and Tenorio, F., Screening for cervical cancer in Latin America: A case-control study. Int. J. Epidemiol. 21, 1150-1156 (1992).
25. Brinton, L. A., and Fraumeni, J. F. Epidemiology of uterine cervical cancer, J. Chronic Dis. 39, 1051-1065 (1986).

26. Brinton, L. A., Tashima, K. T., Lehman, H. F., Levine, R. S., Mallin, K., Savitz, D. A., Stolley, P. D., and Fraumeni, J. F. Epidemiology of cervical cancer by cell type, Cancer Res. 47, 1706-1711 (1987).

27. Winkelstein, W. Cigarette smoking and cancer of the uterine cervix, Banbury Rep. 23, 329-341 (1986).

28. Brand, E., Berek, J. S., and Hacker, N. F. Controversies in the management of cervical adenocarcinoma, Obstet. Gynecol. 71, 261-269 (1988).

29. Cook, G. A., and Draper, G. J. Trends in cervical cancer and carcinoma in situ in Great Britain. Br. J. Cancer 50, 367-371 (1984).

30. Muir, C., Waterhouse, J., Mack, T., Powell, J., Whelan, S. (Eds.). Cancer incidence in five continents, Vol. V, IARC Scientific Publications, No. 88. International Agency for Research on Cancer, Lyon (1987). 